\title{
Mathematical analysis of the effect of the constant temperature assumption in the leak test of PTB Testing Instructions, Volume 25
}

\author{
Oscar Yazit Salah García, Carlos Eduardo García Sánchez \\ Grupo de Investigación en Fluidos y Energía, Centro de Desarrollo Tecnológico del Gas, Parque Tecnológico Guatiguará (km 2 vía Refugio), \\ Piedecuesta, Colombia
}

\begin{abstract}
"PTB Testing Instructions, Volume 25: Gas meters - Test rigs with critical nozzles" (PTB 25) includes a detailed description of a test to evaluate the tightness of the rig (leak test). The mathematical modeling of this test assumes that temperature remains constant, and it is established a maximum allowable change of $0.1 \mathrm{~K}$ during the test. PTB 25 defines a maximum value of the leak flow rate as criteria for approval of the test.

In this work, the effect of the assumption of constant temperature over the leak test result was analyzed. Different processes in which the real leak flow rate was zero and there was a change in the temperature of the system equal to the maximum permissible (according to PTB 25) were simulated, and the leak flow rate calculated by PTB 25 's model was determined. The assumption of constant temperature does not simplifies drastically the mathematical model. It is concluded that PTB 25's maximum allowable temperature change is too high for most practical situations, leading to errors that exceeds the maximum allowable leakage rate. The factors "test time" and "enclosed volume" have a high effect over the magnitude of the error given by PTB 25's leak flow rate model.
\end{abstract}

Section: RESEARCH PAPER

Keywords: leak flow rate; modelling; temperature; PTB 25

Citation: Oscar Yazit Salah García, Carlos Eduardo García Sánchez, Mathematical analysis of the effect of the constant temperature assumption in the leak test of PTB Testing Instructions, Volume 25, Acta IMEKO, vol. 7, no. 1, article 13, March 2018, identifier: IMEKO-ACTA-07 (2018)-01-13

Section Editor: Jorge Torres-Guzman, CENAM - Centro Nacional de Metrologia, Santiago de Querétaro, Mexico

Received July 13,2017; In final form November 18, 2017; Published March 2018

Copyright: ( 2018 IMEKO. This is an open-access article distributed under the terms of the Creative Commons Attribution 3.0 License, which permits unrestricted use, distribution, and reproduction in any medium, provided the original author and source are credited

Corresponding author: Carlos Eduardo García Sánchez, e-mail: cgarcia@cdtdegas.com

\section{INTRODUCTION}

"PTB Testing Instructions, Volume 25: Gas meters - Test rigs with critical nozzles" (PTB 25) is a document that contains physical fundamentals, mathematical models, and design and operation parameters for sonic nozzle test rigs used for calibration of gas meters [1]. PTB 25 is a very complete text for design, construction and operation of calibration benches with critical nozzles, intended to function with air at pressures close to the atmospheric pressure. For this reason, PTB 25 is used as a reference document by many manufacturers and operators of nozzle test rigs for calibration.

In the section which is dedicated to the use of the nozzle test rig, it is included an assay which is intended to evaluate the tightness of the test section of the rig. This assay will be referred as the "leak test" in the rest of this work. It is assumed in PTB 25 that the leak test is an isothermal process, and this has a significant impact over the resulting equations to evaluate the leak test results.

The goal of the present work is to analyse if the mathematical model of the leak test used in PTB 25, which assumes constant temperature, is adequate for the evaluation of the tightness of a nozzle test rig. In Section 2 the modelling of the leak test process and how the assumption of isothermal process converts the model in the one shown in PTB 25 are showed, and after that the simulations done in the present work are described. In Section 3 the results of the simulations are presented and discussed. Finally, in Section 4 the conclusions of the work are summarized. 


\section{METHODS AND MODELS}

The first step in the leak test consists in generating a pressure differential between the interior and the exterior of the nozzle test rig. The next step is to close the bench valves upstream and downstream the test section, so that the region of interest of the bench is a closed system (i.e. it does not allow transfer of mass). If the rig does not have sufficient airtightness, there will be an air flow from the outside of the rig to the inside (or vice versa, depending on the pressure differential). After a period of stabilization, the initial values of pressure and temperature are registered. Subsequently, a certain test time is allowed, and test's final pressure and temperature are registered. With the recorded data and the test time, it is possible to quantify the leak of substance and to get the conclusions of the test. Figure 1 shows a schematic diagram of the process for the leak test of nozzle test rigs.

Section 2.1 shows the mathematical model for the described process. Section 2.2 presents the constant temperature simplification used in PTB 25, and shows the resulting mathematical model. Section 2.3 describes the case studies used in the present work to investigate the effect of PTB 25's assumption over the leak test.

\subsection{Modelling of the leak test}

In the following, the situation in which internal pressure is lower than external pressure is considered. Units are as follow: mass terms are in $\mathrm{kg}$, volume $V$ is in $\mathrm{m}^{3}$, temperature terms are in $\mathrm{K}$, and $R^{\prime}$ is in $\mathrm{Pa}^{*} \mathrm{~m}^{3 *} \mathrm{~kg}^{-1 *} \mathrm{~K}^{-1}$. Mass balance of the leak test process (shown in Figure 1) leads to equation (1) and (2), where $m_{i}$ is the initial mass of gas in the system, $m_{f}$ is the mass of gas in the system at the end of the process, and $m_{\text {leak }}$ represents the mass entering the system during the process, that is, equals the mass of the leak. [2].

$$
\begin{aligned}
& m_{f}=m_{i}+m_{\text {leak }} \\
& m_{\text {leak }}=m_{f}-m_{i}
\end{aligned}
$$

Pressure in the system is not high, so the ideal gas model can be considered valid. Substituting the aforementioned model in equation (2), the equation (3) is obtained.

$$
m_{\text {leak }}=\frac{P_{f} V}{R_{f}^{\prime} T_{f}}-\frac{P_{i} V}{R_{i}^{\prime} T_{i}}
$$

In equation (3), $R^{\prime}$ is the specific gas constant of the gas or mixture of gases that are enclosed in the control volume. $V$ represents the control volume (enclosed volume), and it is constant (the leak test is an isochoric process). If the gas at the interior of the control volume do not change its chemical composition, the specific gas constant will be the same at the start and at the end of the process, so the equation (4) can be written.

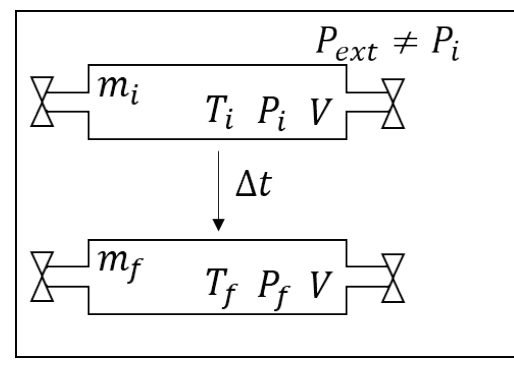

Figure 1. Scheme of the leak test process.

$$
m_{\text {leak }}=\frac{V}{R^{\prime}}\left(\frac{P_{f}}{T_{f}}-\frac{P_{i}}{T_{i}}\right)
$$

Expressing the leak volume in the final test conditions, equation (5) is obtained.

$$
\frac{P_{f} V_{\text {leak }}}{R^{\prime} T_{f}}=\frac{V}{R^{\prime}}\left(\frac{P_{f}}{T_{f}}-\frac{P_{i}}{T_{i}}\right)
$$

Writing the leak volume in term of the mean leak flow rate, $V_{\text {leak }}=Q_{\text {leak }} \Delta t$, where $\Delta t$ is the duration of the leak test and $Q_{\text {leak }}$ is the average flow rate of the leak. Substituting in equation (5), the following equations are developed:

$$
\begin{aligned}
& \frac{P_{f} Q_{\text {leak }} \Delta t}{T_{f}}=V\left(\frac{P_{f}}{T_{f}}-\frac{P_{i}}{T_{i}}\right) \\
& Q_{\text {leak }}=\frac{V T_{f}}{P_{f} \Delta t}\left(\frac{P_{f}}{T_{f}}-\frac{P_{i}}{T_{i}}\right) \\
& Q_{\text {leak }}=\frac{V}{\Delta t}\left(1-\frac{P_{i} T_{f}}{T_{i} P_{f}}\right)
\end{aligned}
$$

If the ideal gas model is valid and assuming temperature homogeneity at the interior of the nozzle test rig, Equation (8) represents correctly the mean leak flow rate during the leak test.

\subsection{Modelling of the leak test in PTB 25}

The leak test model used in PTB 25 to obtain the leak flow rate is based in the mathematical process showed in Section 2.1, but additionally it is assumed that the temperature at the interior of the nozzle test rig does not change throughout the leak test. With the aforementioned assumption, equation (8) is simplified into equations (9) and (10). Equation (10) corresponds to PTB 25's equation (45) [1].

$$
\begin{aligned}
Q_{\text {leak }, \text { PTB25 }} & =\frac{V T}{P_{f} \Delta t}\left(\frac{P_{f}-P_{i}}{T}\right) \\
Q_{\text {leak }, \text { PTB25 }} & =\frac{V}{\Delta t}\left(\frac{\Delta P}{P_{f}}\right)
\end{aligned}
$$

$\Delta P$ is the difference between the final and initial pressure in the leak test. In PTB 25, equation (10) is used in conjunction with a maximum allowable value of leak to give the conclusion of the test.

Equation (10) is correct for isothermal leak tests, modeling the gas mixture as an ideal gas and assuming temperature homogeneity. In the practice, it is almost impossible to guarantee a perfect stability of temperature. For this reason, PTB 25 establishes a non-zero maximum permissible change in the temperature, being equal to $0.1 \mathrm{~K}$. However, temperature has a high effect over the volume of gases, and also the leak flow rates to be identified have very low values. In the present study, it was therefore decided to simulate several different conditions to determine whether the maximum allowable temperature change of PTB 25 ensures the validity of the equation (10), or whether it is necessary to use equation (8) for the quantification of leaks.

PTB 25 establishes an upper limit over the leak value, taken from another volume of PTB Testing Instructions (Volume 4) [1]. This upper limit serves as acceptance criterion, so if the leak rate is lower than the maximum permissible leak rate during the leak test, then the rig is considered airtight. This upper limit is $0.3 \%$ of the minimum flow rate for diaphragm gas meters, and $0.1 \%$ for other kinds of meters. So the maximum permissible leak flow rate for G10 diaphragm meters during the leak test is $8.333 \times 10^{-8} \mathrm{~m}^{3} / \mathrm{s}$; for G16, $1.333 \times 10^{-7} \mathrm{~m}^{3} / \mathrm{s}$; and for G25, $2.083 \times 10^{-7} \mathrm{~m}^{3} / \mathrm{s}$. On the other hand, a non-diaphragm gas meter with a minimum flow rate of $0.10 \mathrm{~m}^{3} / \mathrm{h}$ would have a maximum leak rate of $2.78 \times 10^{-8} \mathrm{~m}^{3} / \mathrm{s}$. 


\subsection{Case studies}

As case studies were used situations in which the rig is airtight (that is, there is no leak), but there is a change of temperature equal to the maximum permitted change according to PTB 25 (that is, $T_{f}=T_{i}+T_{m p c}$ or $T_{f}=T_{i}-T_{m p c}$, where $T_{m p c}$ is $\left.0.1 \mathrm{~K}\right)$. So in all of the simulated situations, actual $Q_{\text {leak }}$ (equation (8)) is zero, but $Q_{\text {leak,PTB25 }}$ (equation (10)) is erroneously not zero.

If there is no leak, the mass balance of the control volume leads to the following relationship between initial and final pressure of the process:

$$
\frac{P_{i} V}{n_{i} R T_{i}}=\frac{P_{f} V}{n_{f} R T_{f}} \rightarrow \frac{P_{i}}{T_{i}}=\frac{P_{f}}{T_{f}} \rightarrow P_{f}=P_{i}\left(\frac{T_{f}}{T_{i}}\right)
$$

In this way, the restrictions imposed over the simulation (no leak, change of temperature of $0.1 \mathrm{~K}$ or $-0.1 \mathrm{~K}$ ) set the value of the final temperature and pressure, and there are four input variables $\left(V, \Delta t, T_{i}\right.$ and $\left.P_{i}\right)$. It was decided to study four levels of every input variable, and test all the possible combinations between the levels of the variables. This study was done two times: the first one with a temperature change of $-0.1 \mathrm{~K}$, and after that with $0.1 \mathrm{~K}$. Thus, there were 256 simulated cases with temperature decrease, and 256 with temperature increase during the leak test. In all cases, $P_{i}$ was lower than the external pressure, so the direction of the leak (if it existed) would be inward of the enclosed volume.

As levels for the input variables were chosen the following sets of values: $V=\{0.025,0.050,0.100,0.150\}$ in $\mathrm{m}^{3} ; \Delta t=\{60$, $120,240,360\}$ in s; $T_{i}=\{280,290,300,310\}$ in $\mathrm{K}$; and $P_{i}=$ $\{70000,80000,90000,100000\}$ in Pa. Those levels represents common values in actual nozzle test rigs.

\section{RESULTS}

In Section 3.1 the results of the case studies with temperature decrease during the leak test are presented. Section 3.2 shows the results of case studies in which an increment of temperature was simulated. Figures 2 to 6 presents the results. The figures include lines showing the maximum leak flow rates $\left(Q_{\text {leak,max }}\right)$, according to PTB 25, in three different situations. The blue line represents $Q_{\text {leak,max }}$ for a rig used to calibrate G10 diaphragm gas meters; the orange one, $Q_{\text {leak,max }}$ for a

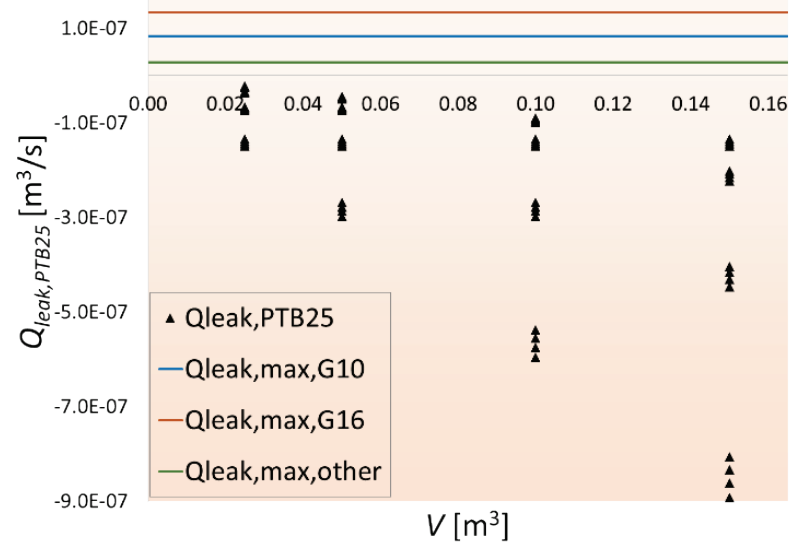

Figure 2. Calculated leak flow rates using PTB 25's model in the simulated cases (leak flow rate is zero) with decreasing temperature in the process $\left(T_{f}=T_{i}-0.1\right)$ versus enclosed volume. The lines show the maximum allowable leak flow rate (according to PTB 25) for calibration rigs for three kinds of meters (details in the text). calibration bench of G16 diaphragm meters; and the green one, $Q_{\text {leak,max }}$ assigned to a calibration rig for gas meters which are not of diaphragm type and have a minimum flow rate of 0.1 $\mathrm{m}^{3} / \mathrm{h}$.

\subsection{Decreasing temperature}

Figure 2 presents $Q_{\text {leak,PTB25 }}$ for the case studies with temperature reduction; in the abscissa the enclosed volume is shown.

In all the cases in which there was simulated a decrease of the temperature equal to the maximum change allowed for PTB 25 , there was a negative error in the calculation of the leak flow rate. In other words, PTB 25's model calculates a negative leak (which is not possible) in the case studies in which $T_{f}=T_{i}-$ 0.1 and there is not leak. This means that a reduction of temperature, even within the limits established in PTB 25, would contribute to conceal the presence of a leak in the control volume when using the mathematical model for leak tests presented in PTB 25. It is also important to notice that the magnitude of the negative leak flow rates is not negligible compared with the allowable leak flow rate.

\subsection{Increasing temperature}

In the case studies with increasing temperature, many values of $Q_{\text {leak,PTB25 }}$ exceed the maximum allowable leak rate, leading to wrong conclusions about the airtightness of the rig. The effect of every one of the four studied factors is analysed. Results are presented using pairwise scatter plots, with $Q_{\text {leak,PTB25 }}$ in the ordinate in all cases, and lines showing maximum permissible leak flow rates, as described at the start of Section 3.

Figure 3 presents the effect of the enclosed volume on the error of PTB 25's leak test model. It is evident that an increase in the enclosed volume raises the error in PTB 25's leak model, and that the effect of the former variable over the latter is very large. The enclosed volume, for the case of critical nozzle test rigs, is a property of the rig which depends mainly of the internal volume of the gas meters that are calibrated in the test rig (which is associated with the kind of gas meter, which in turn affects $Q_{\text {leak,max }}$ ), and of the quantity of gas meters that can be calibrated simultaneously. In almost all of the studied cases, $Q_{\text {leak,PTB25 }}$ exceeded $Q_{\text {leak,max,other }}$.

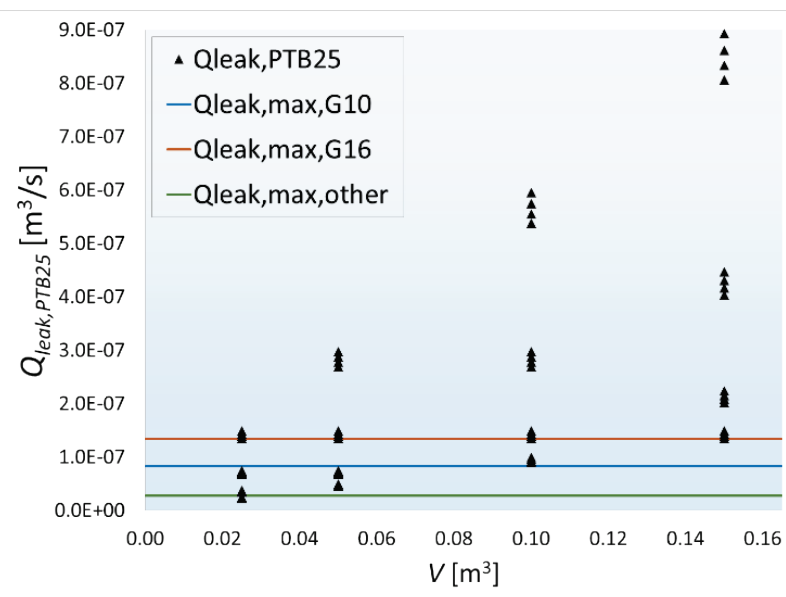

Figure 3. Leak flow rates calculated using PTB 25 's model in cases with temperature increase $\left(T_{f}=T_{i}+0.1\right)$, versus enclosed volume. The lines present the maximum allowable leak flow rate according to the meters that are calibrated in the test rig. 
$Q_{\text {leak,PTB25 }}$ was larger $\tan Q_{\text {leak,max,G10 }}$ for enclosed volumes higher than $0.1 \mathrm{~m}^{3}$, while for smaller enclosed volumes obtaining a correct or incorrect conclusion about the tightness depends on the time elapsed of the leak test. A similar reasoning can be applied to $Q_{\text {leak,max,G16, }}$, although with a little more success regarding the correct conclusion of the leak test.

The effect of the duration of the leak test on PTB 25's error in the calculation of the leak flow rate is presented in Figure 4. An increase in the test time causes a reduction in the error committed by PTB 25's leak flow rate model, so an increase large enough of the test time would make it possible to guarantee that PTB 25's leak test equation yields the correct conclusion. However, it is important to remember that the simulated cases conform to PTB 25's restriction with respect to the maximum permissible change in temperature $(0.1 \mathrm{~K})$. In practice, a longer leak test makes it more difficult to maintain the desired stability in temperature, and additionally a larger duration of the test is undesirable for users of the calibration rigs.

Figure 5 illustrates the relationship between the calculated leak flow rate according to PTB 25 and the initial temperature of the leak test. Figure 6 shows $Q_{\text {leak,PTB25 }}$ versus the initial

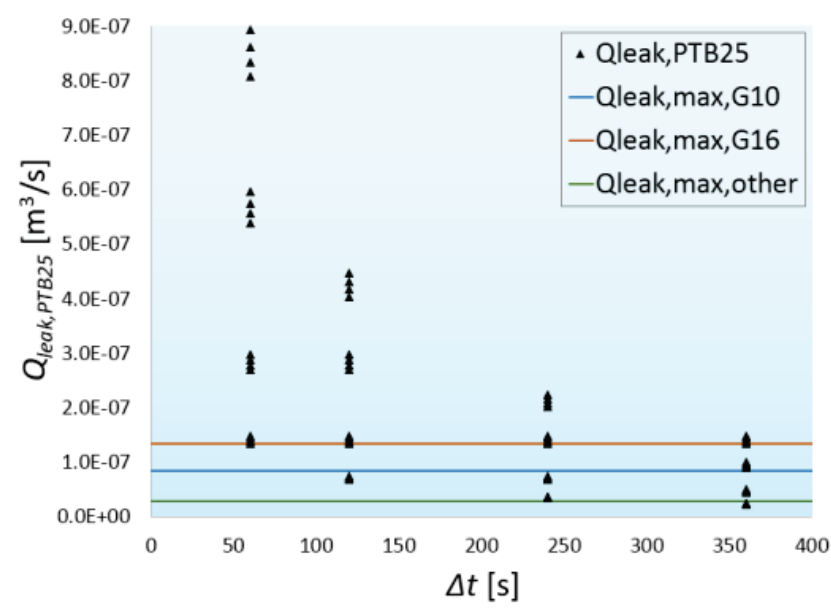

Figure 4. Calculated leak flow rates using PTB 25's model in the cases with temperature increase $\left(T_{f}=T_{i}+0.1\right)$, versus leak test duration. The lines indicate the maximum allowable leak flow rate in three different cases.

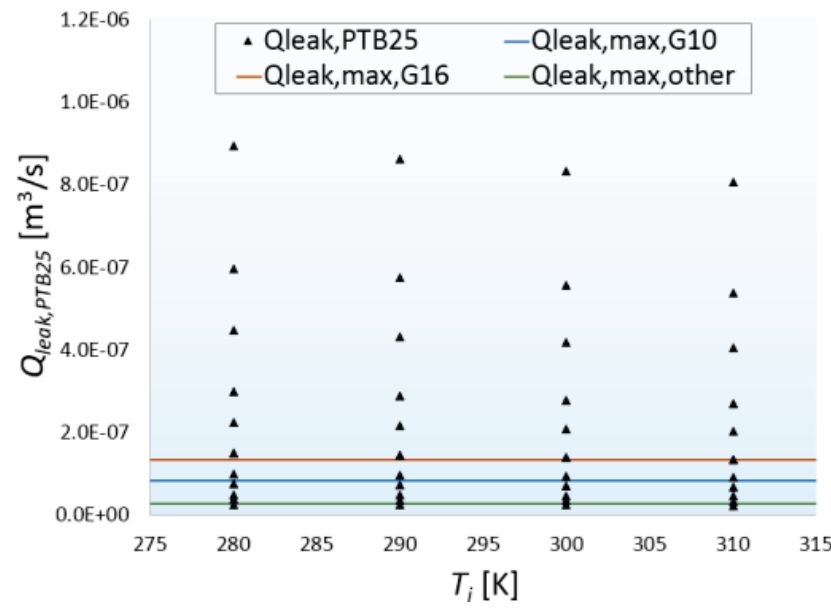

Figure 5. Calculated leak flow rates using PTB $25^{\prime}$ s model in the case studies with temperature increase $\left(T_{f}=T_{i}+0.1\right)$, versus initial temperature in the leak test. The lines indicate the maximum allowable leak flow rate in three different cases. pressure of the enclosed volume in the leak test.

In these last two cases, the effect of the studied factors $\left(T_{i}\right.$ and $P_{i}$ ) on the error of PTB 25's leak flow rate equation is negligible, so neither of these variables could be modified to lessen the error of the equation.

\section{CONCLUSIONS}

In the mathematical modelling of the leak test in PTB 25 it is assumed that the temperature is constant, but the same document allows a temperature change during the experimental run of the test. This causes that the equation used to determine the leak flow rate of the calibration rig has a significant error, in many situations so large that it conduces to erroneous conclusions about the tightness of the rig.

The magnitude of the error committed by PTB 25's leak test equation depends on the enclosed volume of the rig and the duration of the leak test. On the other hand, values of initial pressure and temperature do not affect significantly the error of PTB 25 equation.

The correct modelling of the process of the leak test for sonic nozzle test rigs operating at low pressures leads to the equation (8) of this work. PTB 25 adds the assumption that the process is isothermal, and this leads to equation (10) of the present paper. A comparison of the equations allows to conclude that the difference in complexity is negligible. Additionally, both models require the same measurements; although in equation (10) the initial and final temperatures of the test do not appear, they must also be measured in this case to determine compliance with the restriction on the maximum permissible change in temperature. For these reasons and considering the errors that can be made in estimating the leak flow rate using equation (10), it is possible to conclude that it is preferable to use equation (8) to determine the leak flow rate of a calibration rig in the leak test.

\section{REFERENCES}

[1] G. Wendt, H. Dietrich, B. Jarosch, R. Joest, B. Nath, F. Frössl, M. Ruwe, "PTB Testing Instructions, Volume 25: Gas Meters Test Rigs with Critical Nozzles", Physikalisch-Technische Bundesanstalt, Berlin, 2000, ISSN 0341-7964.

[2] G. V. Reklaitis. "Introduction to Material and Energy Balances", John Wiley and Sons, 1983, ISBN 978-0-471-04131-3.

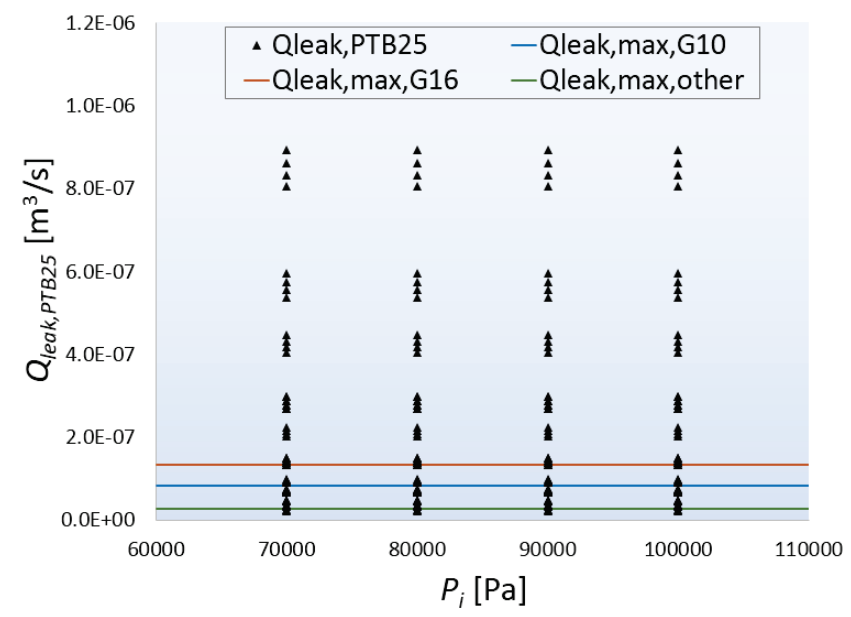

Figure 6. Leak flow rates calculated using PTB 25's model in leak tests with temperature increase $\left(T_{f}=T_{i}+0.1\right)$, versus initial pressure of the control volume. The lines indicate the maximum allowable leak flow rate according to the meters that are calibrated in the test rig. 